\section{Zahl der dokumentierten Melanome nimmt zu}

\author{
Um die Stadienverteilung von Melanomen und die Überlebensraten der \\ Patienten zu analysieren, hatte die Arbeitsgemeinschaft Deutscher \\ Tumorzentren (ADT) die deutschen populationsbasierten und klinischen \\ Tumorregister um Daten zu den zwischen 2002 und 2011 neu diagnostizierten \\ Melanomen gebeten. Nun liegt die Auswertung von 61.895 Fällen vor.
}

E

ner aktuellen Studie zufolge ist die

Zahl der jährlich dokumentierten Melanome zwischen 2002 und 2011 um $53,2 \%$ gewachsen, nämlich von 4.779 auf 7.320. Die Zahl der diagnostizierten Insitu-Melanome sowie Melanome des UICC-Stadiums I wuchs dabei kontinuierlich über die untersuchte Spanne (2002: 612 bzw. 1.939; 2011: 1.625 bzw. 2.990). Die Fälle von UICC-Stadium II gingen hingegen zurück (2002: 814; 2011: 720), wobei der Verlauf seit 2003 relativ konstant war. Melanome im UICC-Stadium III nahmen zu (2002: 260; 2011: 392), und auch im UICC-Stadium IV war ein leichter Zuwachs $\mathrm{zu}$ verzeichnen (2002: 128; 2011: 147).

Insgesamt änderte sich die Stadienverteilung. Der Anteil der In-situ-Melanome stieg von $12,8 \%$ auf $22,9 \%$, jener der Melanome im Stadium I von $61,7 \%$ auf $70,4 \%$. Hingegen fielen die Tumoren im
Stadium II von $25,9 \%$ auf $16,9 \%$. Für die Stadien III und IV war kein statistisch

Die Fünf-Jahres-Überlebensrate für invasiv wachsende Melanome - also ab Stadium I - betrug 83,4\%. Frauen schnitten dabei mit $86,2 \%$ besser ab als Männer mit $80,5 \%$. Auch überlebten mehr jüngere Patienten als ältere. Für die Altersgruppe der 15- bis 34-Jährigen betrug die Überlebensrate 94,6\%, für 65 bis 79 -Jährige und Ältere noch $78,4 \%$. Die Raten des Fünf-Jahres-Überlebens für die einzelnen Stadien lagen bei $96,8 \%$ (I), 74,2\% (II), 56,7\% (III) und $18,4 \%$ (IV). Gezielte Therapien für Patienten in fortgeschritten Stadien waren bis 2011 nicht eingeführt.

Seit Mitte 2008 existiert in Deutschland ein Programm zum Screening auf Hautkrebs, an dem sich etwa $30 \%$ der Berechtigten mit einem Eintrittsalter von relevanter Trend festzustellen.
35 Jahren beteiligen. Aus den Daten, die von der ADT im Zuge der Studie erhoben worden sind, ist ein Effekt des Screenings bis zum Jahr 2011 nicht abzulesen. Die Veränderungen der Fallzahlen zwischen 2002 und 2011 verliefen stetig, und es gab keine ausgeprägten proportionalen Veränderungen in der Stadienverteilung. Zwar waren im Screening entdeckte Melanome mit einer höheren FünfJahres-Überlebensrate verknüpft (94,2\% vs. $84,1 \%$ für die übrigen Fälle). Die Autoren wollen aber nicht ausschließen, dass sich hier systematische Verzerrungen auswirken, etwa ein Selektions- oder auch ein Lead-Time-Bias. Die Daten erlauben es demnach nicht, schlüssig zu belegen, dass das Hautkrebsscreening sich positiv auf das Überleben auswirkt.

Fazit: Von 2002 bis 2011 ist die Zahl der jährlich dokumentierten Melanomfälle in Deutschland um mehr als die Hälfte gewachsen. Dabei hat sich auch die Stadienverteilung geändert, ohne dass dies aber einem Screeningeffekt zugeordnet werden könnte.

Dr. Robert Bublak

Schoffer $\mathrm{O}$ et al. Tumour stage distribution and survival of malignant melanoma in Germany 2002-2011. BMC Cancer 2016; 16: 936

\title{
Dieses Geschwür ist alles andere als banal
}

Eine 73-jährige Frau war über einen weißen Fleck und ein kleines Geschwür am rechten Zungenrand, die seit etwa einem Jahr bestanden, beunruhigt. Beim Essen empfand sie leichte Schmerzen an der Stelle. Sie war Nichtraucherin und trank keinen Alkohol. Bei der körperlichen Untersuchung waren keine vergrößerten Lymphknoten feststellbar. Am rechten Zungenrand fand sich ein $7 \times 3$ $\mathrm{mm}$ großes Ulkus mit glattem Grund, induriertem Rand und fleckig geröteter Umgebung (Abb. a). Die Biopsie ergab \section{ein invasives Plattenepithelkarzinom (Abb. b). Eine Biopsie aus der Stelle mit fleckiger Rötung zeigte hyperchromatische und} polymorphe Zellen der Basalmembran. Dieser Befund entsprach einer leichten Dysplasie (Abb. C). Computertomografisch ergaben sich keine Hinweise auf eine Beteiligung von Halslymphknoten. Nach einer Exzision weit im Gesunden wies die Patientin bei einer Nachuntersuchung ein Jahr später keine Hinweise für ein Rezidiv auf.

Die Fünf-Jahres-Überlebensrate von Patienten mit Plattenepithelkarzinomen der Zunge hat sich in den letzten Jahren trotz des medizinischen Fortschritts kaum verändert und liegt bei deprimierenden $50 \%$. Dies liegt wahrscheinlich an der späten Diagnosestellung, da Ulzerationen und Verhärtungen im Bereich der Zunge von den Patienten lange bagatellisiert werden und keinen Anlass zu einem Arztbesuch geben. Der vorliegende Fall belegt den Wert mehrerer Biopsien an verschiedenen, unterschiedlich aussehenden Stellen, da die Ausdehnung des Karzinoms makroskopisch nur schwer festzustellen ist.
Prof. Hermann S. FüeßI 